\title{
Slow chaos in surface flows
}

\author{
Corinna Ulcigrai ${ }^{1}$
}

Received: 22 June 2020 / Accepted: 10 October 2020 / Published online: 25 November 2020

(c) The Author(s) 2020

\begin{abstract}
Flows on surfaces describe many systems of physical origin and are one of the most fundamental examples of dynamical systems, studied since Poincará. In the last decade, there have been a lot of advances in our understanding of the chaotic properties of smooth areapreserving flows (a class which include locally Hamiltonian flows), thanks to the connection to Teichmueller dynamics and, very recently, to the influence of the work of Marina Ratner in homogeneous dynamics. We motivate and survey some of the recent breakthroughs on their mixing and spectral properties and the mechanisms, such as shearing, on which they are based, which exploit analytic, arithmetic and geometric techniques.
\end{abstract}

\section{Slowly chaotic dynamical systems}

\section{Deterministic chaos and the butterfly effect}

Dynamical systems provide mathematical models of systems which evolve in time. Many systems phenomena in our world, from the evolution of the weather to the motion of an electron in a metal, can be described by a dynamical system. While in a model one can include a random component, or external noise, we will restrict ourselves to fully deterministic systems, whose evolution is completely described by a system of pre-determined rules or equations. We will furthermore consider continuous time dynamical systems, namely systems for which the time variable is a real parameter $t \in \mathbb{R}$, described by aflow on a space $X$, namely a 1-parameter group ${ }^{1} \varphi_{\mathbb{R}}=\left(\varphi_{t}\right)_{t \in \mathbb{R}}$ of maps $\varphi_{\mathbb{R}}: X \rightarrow X$ (diffeomorphisms if $X$ is a smooth manifold).

Deterministic dynamical systems often display chaotic features (see Sect. 2 for examples), which make their behaviour as time grows hard to predict. This is a phenomenon known as deterministic chaos. One of the best known features of chaotic behaviour is sensitive

\footnotetext{
1 Assuming that $\varphi_{\mathbb{R}}=\left(\varphi_{t}\right)_{t \in \mathbb{R}}$ is a group of diffeomorphisms under composition is equivalent to requiring that $\varphi_{t+s}(x)=\varphi_{t}\left(\varphi_{s}(x)\right)$ for every $x \in X$ (or almost every in the measure-preserving set up introduced below) and every $t, s \in \mathbb{R}$. The typical example of a flow is given by solutions to differential equations. The precise definition of the type of flows on which we will focus here, namely area-preserving flows on a surface, will be given below.
}

\section{Corinna Ulcigrai}

corinna.ulcigrai@math.uzh.ch

1 XXI Congresso dell'Unione Matematica Italiana, Pavia, Italy 
dependence on initial conditions (SDIC for short), a property which was popularized as the butterfly effect. In a system which displays SDIC, a small variation of the initial condition can lead to a macroscopically very different evolution after a long time. In particular, given a flow $\varphi_{\mathbb{R}}: X \rightarrow X$ on a metric space $(X, d)$ with SFIC and a point $x \in X$ (the initial condition) one can find arbitrarily close initial conditions $y \in X$ such that the (forward) trajectories of $x$ and $y$, namely the orbits $\left(\varphi_{t}(x)\right)_{t \geq 0}$ and $\left(\varphi_{t}(y)\right)_{t \geq 0}$ drift apart. $^{2}$

\section{Fast chaos versus slow chaos}

Dynamical systems can roughly be divided in three categories (hyperbolic, elliptic and parabolic) according to the speed of divergence (if any) of close orbits. In a hyperbolic flow, the orbits $\left(\varphi_{t}(x)\right)_{t \geq 0},\left(\varphi_{t}(y)\right)_{t \geq 0}$ of most $^{3}$ pairs $x, y$ of initial conditions diverge exponentially in time (i.e. the distance $d\left(\varphi_{t}(x), \varphi_{t}(y)\right)$, for small values ${ }^{4}$ of time, is described by an exponential function of time. In a parabolic dynamical system, there is also divergence of (most) nearby orbits, but this divergence happens at subexponential (usually polynomial) speed. Finally while the flow is called elliptic if there is no divergence (or perhaps it is slower than polynomial). Thus, both hyperbolic system and parabolic systems display SDIC, but the butterfly effect happens at different speeds (respectively exponentially or (sub)polynomially). We colloquially call these systems respectively fast chaotic (when the butterfly effect is fast, i.e. exponential) and slowly chaotic (when the butterfly effect is slow, i.e. polynomial or slower than polynomial).

While there is a classical and well-developed theory of hyperbolic systems (starting with the study of uniformely hyperbolic dynamical systems, which was already developed in the 1970s by mathematicians such as Anosov and Sinai, Abel Prize in 2014, among others) and also a systematic study of elliptic ones (starting with the theory of circle diffeomorphisms, whose study is intertwined with Hamiltonian dynamics and KAM theory), there is no general theory which describes the dynamics of parabolic flows and only classical and isolated examples are well-understood.

\section{Examples of parabolic systems}

Slowly chaotic (or parabolic) systems include many dynamical systems of interest in physics, such as the Novikov model of electrons in a metal (which will be discussed below), or the Ehrenfest model (also called windtree model) proposed by Paul and Tatjiana Ehrenfest in 1912 to understand thermodynamics laws.

Among examples arising in mathematics, perhaps the most studied (and better understoood) example of a parabolic flow is given in the context of hyperbolic geometry by the

\footnotetext{
${ }^{2}$ More precisely, if $(X, d)$ is a metric space and $\varphi_{\mathbb{R}}: X \rightarrow X$ a continuous flow, $\varphi_{\mathbb{R}}$ has SDIC with sensitivity constant $v>0$ iff for every $x \in X$ and $\epsilon>0$ there exists $y$ such that $d(x, y)<\epsilon$ and there exists $t_{0}=t_{0}(v, y)$ such that $d\left(\varphi_{t_{0}}(x), \varphi_{t_{0}}(y)\right) \geq v$.

${ }^{3}$ Most means here almost every with respect to a natural invariant measure for the flow. The formal definition of a (smooth) hyperbolic flow (on the tangent space to a differentialble manifold) involve the existence of stable and unstable manifolds in the tangent space; the exceptional points $y$ whose trajectories do not diverge from (and actually converge to) the trajectory of a given $x$ form the so-called stable manifold, which has positive codimension in the ambient space.

4 If the space $X$ if compact, the distance between two points has clearly an upped bound, so all statements about divergence must be interpreted infinitesimally, i.e. make sense for of $t$ small, as asymptotic statements as the distance between $y$ and $x$ goes to zero, so that longer times can be considered.
} 
horocycle flow on (the unit tangent bundle of) a compact negatively curved surface: ${ }^{5}$ while the geodesic flow (whose trajectories move along geodesics for the hyperbolic metric) is a classical example of fast chaos and hyperbolic dynamics, when moving along horocycles (which, in the upper half plane $\mathbb{H}$ are circles tangent to the real axis) one can show that divergence of nearbly trajectories is only a quadratic function of time, thus giving slowly chaotic dynamics.

Another fundamental class of homogeneous flows is given by nilflows, or flows on (compact) quotients of nilpotent Lie groups (nilmanifolds); The prototype example in this class are Heisenberg nilflows, given by the action (by left multiplication) of a 1 parameter subgroup of transformations of a compact quotient of the Heisenberg group. ${ }^{6}$

In this survey we will focus on an another fundamental class of parabolic flows, in the context of area-preserving flows on (higher genus) surfaces, given by locally Hamiltonian flows, which are smooth flows which preserve a smooth area-form. The definition is given later on in Sect. 3.2.

Finally, starting from the classical examples of parabolic flows mentioned above, one can build new parabolic flows by considering perturbations: the simplest perturbations are time-changes (or time-reparametrizations) of a given flow, i.e. flows that move points along the same orbits, but with different speed. More precisely, a flow $\tilde{\varphi}_{\mathbb{R}}$ is called a (smooth) time-change of a flow $\varphi_{\mathbb{R}}$ on $X$ if for all $x \in X$ and $t \in \mathbb{R}$ we have $\varphi_{t}(x)=\varphi_{\tau(x, t)}(x)$ for some measurable (smooth) function $\tau: X \times \mathbb{R} \rightarrow \mathbb{R}$. Notice that it follows from this definition that the time-change $\tilde{\varphi}_{\mathbb{R}}$ has exactly the same trajectories than $\varphi_{\mathbb{R}}$ (but the motion along the trajectory has different speed). Some time-changes, known as (smoothly) trivial, give rise to flows that are (smoothly) conjugated (i.e. isomorphic as dynamical systems) to the original one and therefore have the same chaotic properties. A feature of parabolic dynamical systems, though, is that among smooth time-changes, smoothly trivial time-changes are rare, i.e. they often form a finite or countable codimension subspace. ${ }^{7}$ Therefore, the study of non trivial time-changes allows to systematically produce new classes of parabolic flows.

\section{Chaotic properties}

A natural and fundamental question in parabolic dynamics (and dynamics in general) is which chaotic properties -in particular which fine ergodic and spectral properties- are generic among classes of (smooth) slowly chaotic flows. Let us give some examples in this section of which

\footnotetext{
5 In the context of homogeneous dynamics (actions given by group multiplication on quotients of Lie groups), parabolic flows coincide with unipotent flows. Horocycle flows can be seen as the simplest example of unipotent flows on semi-simple Lie groups, given by the left action of upper triangular unipotent matrices in $\operatorname{SL}(2, \mathbb{R})$ on compact (or finite volume) quotients $\operatorname{SL}(2, \mathbb{R}) / \Gamma$.

6 Let us recall that the Heisenberg group can be seen as the group $H$ of $3 \times 3$ upper triangular matrices; a compact Heisenberg nilmanifold is obtained taking the quotient $H / \Gamma$ where $\Gamma<H$ is a discrete subgroup (for example the subgroup of matrices of the same form, but with integer entries). A nilflow on a Heisenberg nilmanifold is then given by the action of a 1-parameter subgroup $\left(h_{t}\right)_{t \in \mathbb{R}} \subset H$ by left multiplication $(g, t) \mapsto h_{t} g$.

7 This is the consequence of the existence of distributional obstructions (invariant distributions) to solve the cohomological equation. The first complete study of this phenomenon is perhaps Katok's work [47,48] on linear skew-shifts of the 2-torus, which are closely related to Heisenberg nilflows. Let us also remark that finitely many invariant distributions for horocycle flows in the finite area, non-compact case were first constructed by Sarnak [76] by methods based on Eisenstein series. The structure of the space of obstructions was described in the case of translation flows (and locally Hamiltonian flows on surfaces) in [27], for nilflows in [24] and for horocycle flows in [22].
} 
type of chaotic properties one might be interested in. We will comment on possible notions of generic in Sect. 3.5.

Different type of chaotic properties are the focus of different branches of dynamics: properties of topological nature (such as existence of dense trajectories) are studied in topological dynamics, measure-theoretical features (such as equidistribution of a trajectory with respect to an equilibrium measure) in ergodic theory, and properties of spectral nature in spectral theory of dynamical systems.

\section{Topological dynamics}

Focus on the most basic questions about the behaviour of trajectories, such as existence and abundance of periodic trajectories (i.e. trajectories of a point $x$ such that there exists a period $t_{0}$ for which $\varphi_{t+t_{0}}(x)=\varphi_{t}(x)$ for all $\left.t \in \mathbb{R}\right)$, or existence and abundance of trajectories which are dense in $X$. A (continuous) flow $\varphi_{\mathbb{R}}: X \rightarrow X$ (where $X$ is a topological- or metric-space) is called minimal if every orbit is dense. In presence of fixed points (which is the case for surface flows in higher genus, which always have singularities for $g \geq 2$ ), the definition of minimality is slightly different: we only require all regular orbits, namely all orbits $\varphi_{\mathbb{R}}(x)$ which are neither a fixed point nor a saddle separatrix, to be dense.

\section{Ergodic theory}

Studies flows which preserve a measure: we assume hence that $(X, \mu)$ is a measure space and that $\varphi_{\mathbb{R}}: X \rightarrow X$ preserves the measure $\mu$, namely for any $A$ measurable set, $\mu(A)=$ $\mu\left(\varphi_{t}(A)\right.$ for all $t \in \mathbb{R}$. If a trajectory is dense, one can further ask whether it is equidistributed with respect to the invariant measure $\mu$, namely if the time spent in a measurable set $A$ is proportional (asymptotically) to its measure $\mu(A)$, or, in formulas, whether

$$
\lim _{T \rightarrow \infty} \frac{1}{T} \int_{0}^{T} \chi_{A}\left(\varphi_{t}(x)\right) \mathrm{d} t=\mu(A)
$$

Systems for which this is true for almost every initial condition $x$ (with respect to $\mu$ ) are ergodic. ${ }^{8}$ A stronger conclusion, namely that equidistribution holds for every point $x \in X$ with an infinite trajectoriy, holds for smooth flows which are uniquely ergodic. ${ }^{9}$

A stronger property, mixing, guarantees equidistributions not only of individual orbits, but of sets pushed under the flow $\varphi_{\mathbb{R}}$ : in a mixing system, every measurable set $A \subset X$ equidistributes (with respect to $\mu$ ) under the flow, i.e.

$$
\lim _{t \rightarrow \infty} \mu\left(\varphi_{t}(A) \cap B\right)=\mu(A) \mu(B)
$$

\footnotetext{
${ }^{8}$ Ergodicity is often defined in terms of metric indecomposability: a measure preserving flow $\varphi_{\mathbb{R}}: X \rightarrow X$ on $(X, \mu)$ is ergodic if any measureable $A$ which is invariant under $\varphi_{\mathbb{R}}$, i.e. such that $\varphi_{t}(A)=A$ for all $t \in \mathbb{R}$, is measure-theoretically trivial, i.e. either $\mu(A)=0$ or $\mu(X \backslash A)=0$. The equivalence of this definition with the equidistribution property of almost every trajectory when $\mu(X)$ is finite is the content of the celebrated Birkhoff ergodic theorem.

${ }^{9}$ Unique ergodicity is usually defined for a topological dynamical system, namely for continuous maps or flows on a topological space $X$. We say that the system is uniquely ergodic if there exists an unique probability measure. One can show that in this case, for observables $f: X \rightarrow \mathbb{R}$ which are continuous, one gets a stronger conclusion that the Birkohff ergodic theorem, since one can show that (1), with $\chi_{A}$ replaced by $f$, holds for every (and not only almost every) initial condition $x \in X$. This definition can be applied to the area-preserving flows which we consider later modulo some technicalities (in particular one has to exclude singularities) and guarantees that all trajectories of non-singular points $x$ whose forward trajectory $\left(\varphi_{t}(x)\right)_{t \geq 0}$ is not a separatrix are equidistributed in the sense that (1) holds for all measurable sets $A \subset S$.
} 
for every measurable set $B$. This property is equivalent to decay of correlations, i.e. for every two smooth observables $f, g: X \rightarrow \mathbb{R}$,

$$
\lim _{t \rightarrow \infty} \int_{X}\left(f \circ \varphi_{t}\right) g \mathrm{~d} \mu-\int_{X} f \mathrm{~d} \mu \int_{X} g \mathrm{~d} \mu=0 .
$$

This property is also known as strong mixing, to distinguish it from another (weaker) property known as weak mixing (where the convergence in (2) is only required to happen along a subset of $t \in \mathbb{R}$ of density one). Mixing and weak mixing can also be interpreted as spectral properties, (see footnote 11). Other type of mixing properties in addition to weak mixing and strong mixing include mild mixing and mixing of all orders. The latter generalizes mixing (which is defined using two sets $A, B)$ to more sets: a measure preserving flow $\varphi_{\mathbb{R}}$ on $(X, \mu)$ is mixing of order $N$ if, for any $N$-tuple $A_{0}, \ldots, A_{N-1}$ of measurables sets,

$$
\begin{aligned}
& \mu\left(A_{0} \cap \varphi_{t_{1}}\left(A_{1}\right) \cap \varphi_{t_{1}+t_{2}}\left(A_{2}\right) \cap \cdots \cap \varphi_{t_{1}+\cdots+t_{N-1}}\left(A_{N-1}\right)\right) \stackrel{t_{1}, t_{2} \ldots, t_{N-1} \rightarrow \infty}{\longrightarrow} \\
& \mu\left(A_{0}\right) \cdots \mu\left(A_{N-1}\right)
\end{aligned}
$$

and it is mixing of all orders if it is $N$-mixing for any $N \geq 2$. Equivalently, as for the definition of mixing, this can be reinterpreted as a statement about decay of multi-correlations. It is a famous open conjecture, known as Rohlin's conjecture and still open, whether mixing implies mixing of all orders.

\section{Spectral theory of dynamical systems}

Study the nature of the spectrum (and spectral measures) associated to the Koopman operator a (family of) operator(s) on $L^{2}(X, \mathscr{A}, \mu)$ associated to measure preserving flow $\varphi_{\mathbb{R}}$ (which acts by pre-composition $f \mapsto f \circ \varphi_{t}$ with the dynamics). One of the fundamental questions in spectral theory (see for example the surveys [49] or [58] on spectral theory of dynamical systems) is what is the nature of the spectrum of the Koopman operator. To every $f \in$ $L^{2}(X, \mu)$ one can associate a spectral measure denoted by $\sigma_{f}$, i.e. the unique finite Borel measure on $\mathbb{R}$ whose Fourier coefficients are described by correlations, i.e. such that

$$
\int_{X} f \circ \varphi_{t} \bar{f} d \mu=\int_{\mathbb{R}} e^{i t s} d \sigma_{g}(s) \text { for every } t \in \mathbb{R} .
$$

Spectral measures are useful to describe components of the the unitary representation given by the Koopman operator. ${ }^{10}$ We say that the spectrum of $\varphi_{\mathbb{R}}$ is (absolutely) continuous, or respectively (purely) singular iff for every $f \in L^{2}(X, \mu)$ the spectral measure $\sigma_{f}$ is (absolutely) continous, or respectively singular with respect to the Lebesgue measure on $\mathbb{R}$.

Ergodicity, weak mixing and mixing can be expressed in terms of the spectrum of the Koopman operator. ${ }^{11}$ Spectral results thus provide finer and stronger dynamical information. For example, since mixing (and weak mixing), when they hold, provide, as spectral implication, the information that the spectrum is continuous (see footnote 11), proving that the spectrum is absolutely continuous is e. g. a strengthening of mixing, while singularity of the

\footnotetext{
$\overline{{ }^{10} \text { Let us denote by } \mathbb{R}(g) \subset L^{2}}(X, \mu)$ the cyclic subspace generated by $g$ which is given by $\mathbb{R}(g):=$ $\overline{\left\langle\varphi_{t}(g): t \in \mathbb{R}\right\rangle} \subset L^{2}(X, \mu)$. By the spectral theorem the Koopman operator, restricted to $\mathbb{R}(g)$, is unitarily isomorphic to the $\mathbb{R}$-representation $\left(V_{t}\right)_{t \in \mathbb{R}}$ on $L^{2}\left(\mathbb{R}, \sigma_{g}\right)$ given by $V_{t}(h)(s)=e^{i t s} h(s)$.

11 Ergodicity is equivalent to asking that the only eigenfunctions for the Koopman operator with eigenvalue 1 are constant functions, while weak mixing holds iff the Koopman operator has no non-constant eigenfunctions (i.e. no eigenvalues different than 1). Hence weak mixing implies that the spectrum is continous; mixing on the other hand is characterized by decay of the self-correlations $\left\langle U_{t} f, f\right\rangle_{L^{2}(X, \mu)}$, see (3).
} 
spectrum shows that the system studied is far (more formally, spectrally disjoint, a stronger concept of disjointess than that introduced in Sect. 3.13) from strongly, fast chaotic systems.

\subsection{Generic chaotic properties in slowly chaotic systems}

There is a large and quite extensive literature on topological, ergodic and spectral properties of some of the classical parabolic examples mentioned in Sect. 1. For example, the fine ergodic and spectral properties of the horocycle flow have been studied in great detail ${ }^{12}$ and mostly were already well understood in the 1970s (see for example [12,22,29,36,57,66,81] and more in general [80] or [2], and the reference therein, for unipotent flows).

It is well known that the typical ${ }^{13}$ nilflow is minimal and uniquely ergodic [2], however, nilflows are never (weak) mixing, due to an intrinsic obstruction, namely the presence of a toral factor. ${ }^{14}$ Results on the speed of equidistribution of Heisenberg nilflows for smooth functions were proved by Flaminio and Forni [23].

A series of recent works $[4,5,74]$ indicates that, even though classical nilflows are never mixing (see footnote 14), a typical time-change (in a dense class of smooth time-changes) of a mimimal nilflow on any nilmanifold (different from a torus) is mixing.

In the rest of this survey we will focus on generic chaotic properties of smooth area preserving flows. While the understanding of minimality and ergodicity follows from results from the 1970s and 1980s respectively, the study of mixing properties has been the object of active research in the last decade or so, while the first breakthroughs on spectral properties are only very recent.

These results do not show an entirely coherent picture and make the identification and description of characteristic parabolic features difficult. For example, while the horocycle flow (as well as all its smooth time-changes) are mixing (and actually mixing of all orders, see $[61,62]$ ), as we recalled above (see in particular footnote 14) nilflows are never mixing. This difference in behavior can be attributed to the lack of parabolicity in certain directions (those that live in the toral factor, see foonote 13), which would suggest calling nilflows partially parabolic systems. Nevertheless, this obstruction can be broken by a perturbation (as we show in [5], see also [4] for the special case of Heisenberg nilflows), so that in a dense set of smooth-time changes all flows which are not trivially conjugate to the nilflow itself are indeed mixing. Similarly, recent results seem to indicate that certain disjointess properties

\footnotetext{
12 For compact hyperbolic surfaces, it is for example known that the horocycle flow is minimal [39], uniquely ergodic [36], mixing [66] (in fact it has Lebesgue spectrum [80] and it is mixing of all orders [62]) and precise bounds on both the rate of mixing [72] and equidistribution [22] are available. For the case of finite-volume, non-compact surfaces, see e.g. the works by Dani [15] and Burger [12].

13 Typical means here for a full measure set of frequencies of the underlying toral factor, defined as follows. Every nilmanifold $G / \Gamma$ is a fiber bundle over a torus. In fact, the group $\bar{G}=[G, G] \backslash G$ is Abelian, connected and simply connected, hence isomorphic to $\mathbb{R}^{n}$ and $\bar{\Gamma}=[\Gamma, \Gamma] \backslash \Gamma$ is a lattice in $\bar{G}$. Thus we have a natural projection $p: G / \Gamma \rightarrow \bar{G} / \bar{\Gamma}$ over a torus of dimension $n$. The nilflow hence project to a linear flow on an $n$-dimensional torus. We say that a property holds for a typical nilflows if it holds for a typical linear flow on the underlying toral factor, namely for a full measure set of rotation numbers (also called frequencies).

14 As explained in the foonote 13 above, any nilflow project on a linear flow on a toral factor. It follows that a nilflow is never weakly mixing (and hence never mixing), since the linear toral flow has pure point spectrum and hence many non-trivial eigenfunctions (the toral characters), which can be pulled back to the nilmanifold to produce eigenfunctions for the nilflow (recall that weak mixing can be characterized in terms of lack of eigenfunctions, see Sect. 2). However, it is possible to prove by methods of representation theory that any nilflow is relatively mixing, in the sense that the limit of correlations of functions with zero average along all fibers of the projection $p$ is equal to zero. Nilflows also have the property of relative Lebesgue spectrum (namely, the spectrum restricted to observables in the orthogonal complement of the span of the pull-back of the toral characters, is countable Lebesgue spectrum, see [2]).
} 
(see Sect. 3.13), which do not hold for a classical example such as the horocycle flow, are nevertheless generic among time-changes.

This shows that to better understand slowly chaotic systems it is therefore crucial both to understand what are the finer chaotic properties of the known parabolic examples as well as, at the same time, to study new classes of parabolic examples (such as those produced by time-changes and other parabolic perturbations. ${ }^{15}$

\section{Chaotic properties of smooth surface flows}

We will focus now on the class of slowly chaotic systems given by smooth area-preserving (or locally Hamiltonian) flows on surfaces, surveying the recent advances in our understanding of their mixing, spectral and disjointness properties as well as the mechanisms which explain them.

\subsection{Flows on surfaces}

Flows on surfaces are one of the most basic and most fundamental examples of dynamical systems, whose study goes back to Poincaré [67] at the end of the Ninetineth century, and coincides with the birth of dynamical systems as a research field. Many models of systems of physical origin are described by surface flows: Poincaré motivation to study surface flows was for example related to celestial mechanics and the two physical systems already mentioned before, the Ehrenfest model in statistical mechanics and the Novikov model in solid state physics, can be described by flows on surfaces (respectively linear flow on an translation surface and to a locally Hamiltonian flows).

In addition to providing a fundamental classes of parabolic dynamical systems, smooth, area-preserving flows on surfaces, are fundamental in dynamics because they are among the lowest possible dimensional smooth dynamical system (on compact manifolds of lower dimension, the other fundamental class of smooth dynamical systems are circle diffeomorphisms, whose rich theory is a cornerstone of dynamics). Despite having zero entropy, as shown in [90], they nevertheless display a rich variety of chaotic properties and, despite their basic nature, there are still many open questions on the mathematical characterization of chaos (in particular on dynamical, spectral and rigidity question) in various natural classes of surface flows.

In this survey we will only be concerned with flows which preserve a (probability) measure (see Sect. 2 for the definition), for example an area-form, since this is the natural setup for ergodic theory (see Sect. 2).

\subsection{Locally Hamiltonian flows}

Let $S$ be a compact, connected, orientable (smooth) surface and let $g$ denote its genus. We will assume throughout that $g \geq 1$. Perhaps the most natural class of measure-preserving

\footnotetext{
15 It should be pointed out that describing more general perturbations (beyond the class of time changes) which produce (new) parabolic flows is quite delicate, since by a perturbation one typically gets a hyperbolic flow. Examples of parabolic perturbations, which are not time changes, can be constructed for example by twisting (see for example the work of Simonelli on twisted horocycle flows, in [79]). New examples of parabolic perturbations for which one can study ergodic theoretical properties were recently constructed by Ravotti in [75].
} 
flows on $S$ are smooth flows preserving a smooth measure (with smooth absolutely continuous density). Let $\omega$ be a fixed smooth area form (locally given in coordinates $(x, y)$ by $f(x, y) d x \wedge$ $d y$ where $f$ is a smooth function). Thus, equivalently, the pair $(S, \omega)$ is a two-dimensional symplectic manifold. We will consider a smooth flow $\varphi_{\mathbb{R}}=\left(\varphi_{t}\right)_{t \in \mathbb{R}}$ on $S$ which preserves a measure $\mu$ given integrating a smooth density with respect to $\omega$. We will assume that the area is normalized so that $\mu(S)=1$. It turns out that such smooth area preserving flows on $S$ are in one-to-one correspondence with smooth closed real-valued differential 1-forms as follows. Given a smooth, closed, real-valued differential 1-form $\eta$, let $X$ be the vector field determined by $\eta=i_{X} \omega$ where $i_{X}$ denotes the contraction operator, i.e. $i_{X} \omega=\omega(\eta, \cdot)$ and consider the flow $\varphi_{\mathbb{R}}$ on $S$ given by $X$. Since $\eta$ is closed, the transformations $\varphi_{t}, t \in \mathbb{R}$, are area-preserving. Conversely, every smooth area-preserving flow can be obtained in this way.

The flow $\varphi_{\mathbb{R}}$ is known as the multi-valued Hamiltonian flow associated to $\eta$. Indeed, the flow $\varphi_{\mathbb{R}}$ is locally Hamiltonian, i.e. locally one can find coordinates $(x, y)$ on $S$ in which $\varphi_{\mathbb{R}}$ is given by the solution to the equations

$$
\left\{\begin{array}{l}
\dot{x}=\partial H / \partial y, \\
\dot{y}=-\partial H / \partial x
\end{array}\right.
$$

for some smooth real-valued Hamiltonian function $H$. A global Hamiltonian $H$ cannot be in general be defined (see [64, Section 1.3.4]), but one can think of $\varphi_{\mathbb{R}}$ as globally given by a multi-valued Hamiltonian function.

Let us remark that locally Hamiltonian flows necessarily have fixed points, or singularities, if $g \geq 2$. Singularities, as shown in Fig. 2, can be either centers (Fig. 2a), simple saddles (Fig. 2b) or multi-saddles (i.e. saddles with $2 k$ pronges, $k \geq 2$, see Fig. $2 \mathrm{c}$ for $k=3$ ), . Examples of flow trajectories are shown in Fig. 1. For $g=1$, i.e. on a torus, if there is a singularity than there has to be another one. The simplest examples of locally Hamiltonian

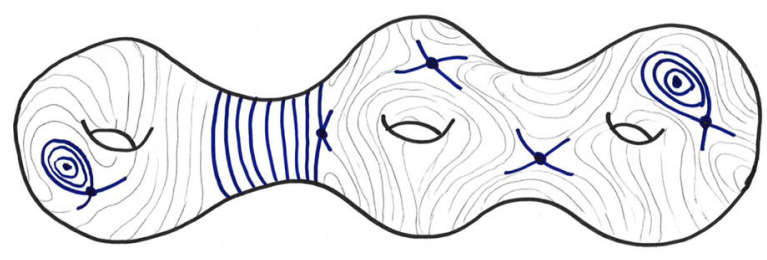

(a) A flow on a surface of $(\mathrm{g}=3)$

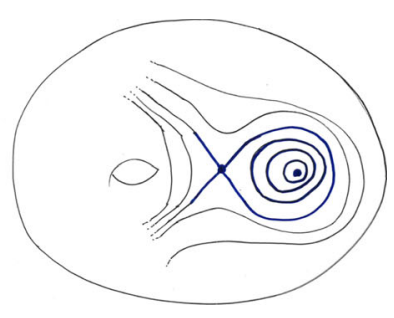

(b) An Arnold flow $(\mathrm{g}=1)$

Fig. 1 Trajectories of locally Hamiltonian flows on a surfaces

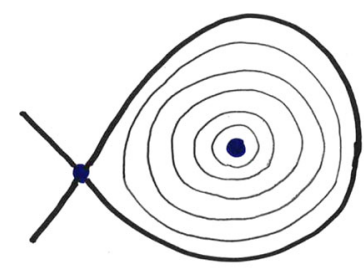

(a) center

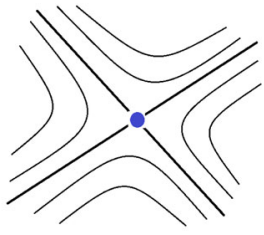

(b) simple saddle

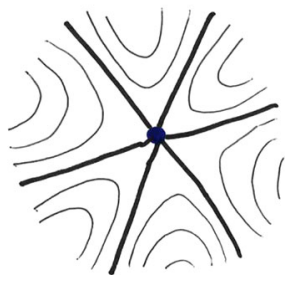

(c)

Fig. 2 Type of singularities (i.e. fixed points) of a locally Hamiltonian flow 
flows with singularities on a torus, i.e. flows with one center and one simple saddle (see Fig. 1b), were studied by Arnold in [1] and are nowadays often called Arnold flows. ${ }^{16}$

A lot of interest in the study of multi-valued Hamiltonians and the associated flows-in particular, in their ergodic and mixing properties—was sparked by Novikov [65] in connection with problems arising in solid-state physics as well as in pseudo-periodic topology (see e.g. the survey [91] by Zorich). Indeed, Novikov [65] and his school in the 1990s advocated the study of locally Hamiltonian flows as model to describe the motion of an electron in a metal under a magnetic field in the semi-classical approximation (the surface appears here as Fermi energy level surface). Novikov made some conjectures (known as Novikov problem) on the asymptotic behaviour of trajectories of electrons. At the same time, Arnold [1] made a conjecture on mixing for the flows we call today Arnold flows (see footnote 16). This conjecture has been the motivation for a lot of the work on the mixing properties of locally Hamiltonian flows, see the overview given in Sect. 3.8.

In order to survey the current knowledge of chaotic properties of locally Hamiltonian flows, it is useful to first point out their relation with another well studied class of areapreserving flows on surfaces, namely linear flows (which, we stress for the reader, are not smooth surface flows).

\subsection{Linear flows and time-changes of locally Hamiltonian flows}

The basic example of a linear flow is the flow given on the torus $\mathbb{R}^{2} / \mathbb{Z}^{2}$ by solutions of

$$
\left(x^{\prime}(t), y^{\prime}(t)\right)=(\cos \theta, \sin \theta),
$$

which move along at unit speed along (the image in $\mathbb{R}^{2} / \mathbb{Z}^{2}$ of) Euclidean lines. Linear flows (also called translation flows) can be defined more in general on translation surfaces, namely surfaces which are locally Euclidean outside a finite number of conical singularities (with cone angles $2 \pi k, k \in \mathbb{N}$, which produce saddles of the flows with $2 k$ prongs, see Fig. $2 \mathrm{c}$ for $k=3$ ). Notice that these flows preserve a Euclidean area (but are discontinuous flows, since singularities are reached in finite time).

It turns out that every minimal locally Hamiltonian flow on $S$ (as well as the restriction of a locally Hamiltonian flow to one of its minimal components, see Sect. 3.6), in suitably chosen coordinates, are time-changes (or time-reparametrizations, we refer to Sect. 1 for the definition) of a linear flow on $S$ (or a subsurface of $S$ in the case of a minimal component). Thus, minimal locally Hamiltonian flows have the same trajectories (up to time-reparametrization) than linear flows on translation surfaces (see for example [91]). In particular, certain properties, such as minimality and ergodicity (as well as homological aspects such as the asymptotic behaviour in the Novikov problem), which depend only on trajectories as sets and not on their time-parametrization, can be deduced for locally Hamiltonian flows by studying them in linear flows. This was in part one of the original motivations (in addition to unfolding of rational billiards in the West) that sparked the interest of mathematicians such as Zorich in the ergodic theory of linear flows (see below). We stress though that, while some chaotic properties like ergodicity depend on the orbits of the flow, others (like mixing and spectral properties) crucially depend on the time-parametrization of the orbits and require ad-hoc techniques (see Sects. 3.9and 3.14).

\footnotetext{
16 More precisely, referring to the decomposition described in Sect. 3.6, we call Arnold flow the restriction to a minimal component obtained by removing the center and the disk filled by periodic orbits around it (called island), which, as Arnold shows in [1], is always bounded by a saddle loop.
} 


\subsection{Linear flows and Teichmueller dynamics}

The study of linear flows on translation flows and their ergodic properties has been a highly topical area of research for the past four decades (from the 1980s), in connection with the study of billiards in (rational) polygons, interval exchange transformations (or for short IETs) and Teichmueller dynamics, a research area which has benefited from the contribution of several Fields medallists (including Avila, Kontsevich, McMullen, Mirzakhani and Yoccoz).

In virtue of this flourishing activity, the ergodic and spectral properties of typical (in the measure theoretical sense) translation flows are by now well understood. Let us say that a property holds for a typical linear flow if it holds in a.e. direction on almost every translation surface with respect to a natural measure on translation surfaces known MasurVeech measure. ${ }^{17}$ One of the first results, shown already in the 1970s by Keane [50], is that a typical linear flow is minimal. Moreover, it is uniquely ergodic (which implies that every infinite orbit is not only dense, but also equidistributed, see Sect. 2). Unique ergodicity of typical linear flows was known as Keane's conjecture and proved independently in the seminal works by Masur and Veech $[63,87]$ through renormalization techniques which gave birth to the topical field of Teichmueller dynamics. On the other hand linear flows are never mixing, as proved by Katok [46] already in the 1980s, but they are neverthelss typically (in the above sense) weak-mixing (refer to Sect. 2 for definitions). a long-standing conjecture settled by Avila and Forni in [3].

From the spectral theory perspective, for typical translation flows, the nature of the spectrum (which turns out to be singular continuous) has been known since seminal work by Veech, see $[87,88])$. Recently there have been also advances in the spectral theory of non generic (especially self-similar) translation flows and IETs, see for example [9-11].

We now discuss locally Hamiltonian flows, explaining how minimality and unique ergodicity can be understood reducing the study of (minimal components of) locally Hamiltonian flows to linear flows, while the classification of mixing properties (described in Sect. 3.8) has been based on geometric mixing mechanisms specific to smooth slowly parabolic flows (such as shearing, see Sect. 3.9) and the spectral theory is only now starting to be understood (refer to Sect. 3.14). We first specify (in the next Sect. 3.5) the topology and measure that we will use on the space of locally Hamiltonian flows.

\subsection{Genericity notions for locally Hamiltonian flows}

Let us define two natural ways of defining a notion of generic (or typical) locally Hamiltonian flow, one topological and the other measure-theoretical.

One can define a topology on locally Hamiltonian flows by considering perturbations of closed smooth 1-forms by (small) closed smooth 1 -forms. ${ }^{18}$ We say that a condition is generic (in the sense of Baire) if it holds for flows described by an open and dense set of forms with respect to this topology. For example, asking that the 1-form $\eta$ is Morse, i.e. it is locally the differential of a Morse function (which has non-degenerate zeros) is a generic condition.

\footnotetext{
17 Perhaps the simplest way to define the Masur-Veech measure, is to consider a presentation of a translation surface $S$ as a polygon with $2 N$ sides with pairs of parallel, congruent sides $\left(v_{1}, v_{1}^{\prime}\right), \ldots,\left(v_{N}, v_{N}^{\prime}\right)$ identified by glueings. Then the vectors $\left(v_{1}, \ldots, v_{N}\right) \in \mathbb{R}^{N}$ give local coordinates for an open set $U$ of translation surfaces around $S$ and the Masur-Veech measure is just Lebesgue measure on $U \subset \mathbb{R}^{N}$.

18 Let $\eta, \eta^{\prime}$ be two smooth closed 1-forms. We say that $\eta^{\prime}$ is an $\epsilon$-perturbation of $\eta$ if for any $x \in S$ there exists coordinates on a simply connected neighbourhood $U$ of $x$, such that $\eta \|_{U}=d H$ and $\left(\eta^{\prime}-\eta\right) \|_{U}=d h$ where $\|h\|_{\infty}<\epsilon\|H\|_{\infty}$ (here $\|\cdot\| \infty$ denotes the $\mathscr{C}^{\infty}$ norm).
} 
A measure-theoretical notion of typical is defined as follows by using the Katok fundamental class (introduced by Katok in [45], see also [64]), i.e. the cohomology class of the 1-form $\eta$ which defines the flow. Let $\Sigma$ be the set of fixed points of $\eta$ and let $k$ be the cardinality of $\Sigma$. Let $\gamma_{1}, \ldots, \gamma_{n}$ be a base of the relative homology $H_{1}(S, \Sigma, \mathbb{R})$, where $n=2 g+k-1$. The image of $\eta$ by the period map $\operatorname{Per}$ is $\operatorname{Per}(\eta)=\left(\int_{\gamma_{1}} \eta, \ldots, \int_{\gamma_{n}} \eta\right) \in \mathbb{R}^{n}$. The pull-back $P e r_{*} L e b$ of the Lebesgue measure class by the period map gives the desired measure class on closed 1-forms. When we use the expression typical below, we mean full measure with respect to this measure class.

\subsection{Periodic and minimal components}

A generic locally Hamiltonian flow (in the sense of Baire category, with respect to the topology defined in the previous Sect. 3.5) has only non-degenerate fixed points, i.e. centers and simple saddles (see Fig. 2a, b), as opposed to degenerate multi-saddles (as in Fig. 2c). We call saddle connection a flow trajectory from a saddle to a saddle and a saddle loop a saddle connection from a saddle to the same saddle (see Fig. 3a). It can be shown that each center is contained in a disk filled with closed (i.e. periodic) trajectories and bounded by a saddle loop, called an island of periodic orbits, see Fig. 3a. Hence, in presence of centers, the flow $\varphi_{\mathbb{R}}$ is never minimal (since orbits in the complement of the island avoid the island and hence cannot be dense).

From the point of view of topological dynamics (as proved independently by Maier [60], Levitt [59] and Zorich [91]), each smooth area-preserving flow can be decomposed into subsurfaces (with boundary) on which the restriction of $\varphi_{\mathbb{R}}$ either foliates into closed (i.e. periodic) orbits and up to $g$ subsurfaces (recall that $g$ is the genus of $S$ ) on which (the restriction of) $\varphi_{\mathbb{R}}$ is minimal, i.e. every bi-infinite orbit is dense. The first ones are called periodic components and are either islands (as in Fig. 3a) or cylinders filled by periodic orbits and bounded by saddle loops, as in Fig. 3b. The latter are known as minimal components (see an example in Fig. 3c) and by topological reasons there cannot be more than $g$ of them. The flows in Fig. 1, for example, can be decomposed, in the case of Fig. 1a, into two islands and one cylinder filled by closed orbits and two minimal components (one of of genus one and one of genus two), while, in the case of the flow on the torus in Fig. 1b, there is one island and one minimal component (the so-called Arnold flow).

Minimal components of a locally Hamiltonian flow (and in particular minimal such flows, for which $S$ is in itself a minimal component), in suitably chosen coordinates, have the same orbits (up to time-reparametrization, see Sect. 1) than linear flows discussed in Sect. 3.3 (see e.g. [91]).

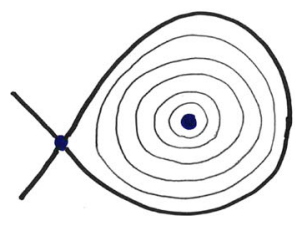

(a) an island of period orbits

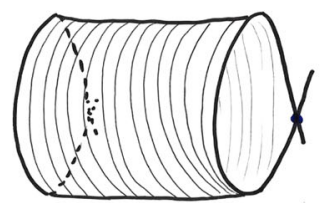

(b) a cylinder of periodic orbits

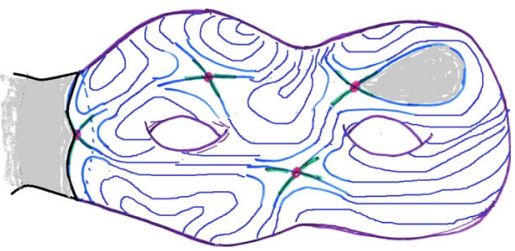

(c) a minimal component of $g=2$

Fig. 3 Examples of periodic and minimal components 


\subsection{Minimality and ergodicity}

To classify chaotic behaviour in locally Hamiltonian flows it is crucial to distinguish between two (complementary, up to measure zero) open sets (with respect to the topology described in Sect. 3.5): in the first open set, which we will denote by $\mathscr{U}_{\text {min }}$, the typical flow is minimal (in particular there are no centers and there is a unique minimal component). On the other open set that we will call $\mathscr{U}_{\neg \min }$ there are periodic components (bounded by saddle loops homologous to zero), but the typical flow is still minimal when restricted to each complementary (minimal) component.

Let us remark that if the flow $\varphi_{\mathbb{R}}$ given by a closed 1 -form $\eta$ has a saddle loop homologous to zero (i.e. the saddle loop is a separating curve on the surface), then the saddle loop is persistent under small perturbations (see Section 2.1 in [91] or Lemma 2.4 in [73]). In particular, the set of locally Hamiltonian flows which have at least one saddle loop is open and gives the set denoted $\mathscr{U}_{\neg \text { min }}$ above. The set $\mathscr{U}_{\text {min }}$ is given by the interior (which one can show to be non-empty) of the complement of $\mathscr{U}_{\neg \min }$, i.e. the set of locally Hamiltonian flows without saddle loops homologous to zero. ${ }^{19}$

The typical locally Hamiltonian flow (with respect to the measure defined in Sect. 3.5) is $\mathscr{U}_{\neg \text { min }}$ is not only minimal, but also uniquely ergodic. For a typical flow in $\mathscr{U}_{\neg \text { min }}$, the restriction of the flow on each minimal component is (uniquely) ergodic. Both results about minimality and ergodicity can be deduced from the classical results respectively by Keane and Masur and Veech (recalled in Sect. 3.4) respectively concerning of minimality and ergodicity of typical translation flows, by using that that the flow restricted to a minimal components is a time-change of a linear flow (see Sect. 3.3). ${ }^{20}$

\subsection{Mixing properties of locally Hamiltonian flows}

As mentioned earlier, finer chaotic properties such as (weak) mixing and spectral properties, crucially depend also on the speed of motion along the orbits.

The question of mixing in locally Hamiltonian flows was motivated by Arnold's conjecture in the 1990s. In contrast with translation flows, which are never mixing (see Sect. 3.4), Arnold in the 1990s noticed a geometric phenomenon (explained in Sect. 3.8) which could produce mixing in locally Hamiltonian flows on the torus with one minimal component (those which we nowadays call Arnold flows, see Sect. 3.6 and in particular footnote 16). His intuition was proved to be correct shortly after by Sinai and Khanin [51], for a full measure set (later improved by Kocergin [53]) of such flows on tori. The question of whether mixing is typical also for flows on higher genus is much more delicate, and stayed open for 2 decades.

It turns out that mixing depends crucially on the type of singularities of the flow. When there are degenerate saddles (i.e. multi saddles with $k \geq 6$ prongs, as in Fig. 2c), mixing had been proved already in the 1970s (by Kochergin in [53]) since in this case the saddles have a much stronger effect. ${ }^{21}$ In the case of non-degenerate saddles (which, we recall, is generic

\footnotetext{
19 Note that saddle loops non homologous to zero (and saddle connections) vanish after arbitrarily small perturbations and neither the set of 1-forms with saddle loops non homologous to zero (or saddle connections) nor its complement is open (see [73] for details).

20 One needs also to exploit that the two notions of typical, respectively for linear flows and locally Hamiltonian flows, interact well with each other: in particular, if a result holds for almost linear flow in almost eveyr direction on almost every translation surface with respect to the Masur-Veech measure, one can show that it holds for a full measure set of locally Hamiltonian flows with respect to the Katok fundamental class.

21 As explained in Sect. 3.9 mixing happens through shearing of transversal arcs and equidistribution of flow trajectories. In the case of degenerate saddles, the shearing effect is much faster and allows for simpler
} 
case, but much more delicate to treat), one has very different results in the open sets $\mathscr{U}_{\text {min }}$ and $\mathscr{U}_{\neg \text { min }}$ introduced in the previous Sect. 3.7. The full classification of mixing properties has been a central part of my past research achievements [84-86]. The two following two results now give a complete picture:

Theorem 1 (U' $[85,86]^{22}$ )

In $\mathscr{U}_{\text {min }}$, the typical locally Hamiltonian flow is weakly mixing, but it is not mixing.

Theorem 2 (U' [84], Ravotti [73] ${ }^{23}$ ) In $\mathscr{U}_{\neg \text { min }}$, the restriction of the typical locally Hamiltonian flow $\varphi_{\mathbb{R}}$ on each of its minimal components is mixing.

Let us remark even though the typical flow in $\mathscr{U}_{\text {min }}$ is not mixing, there exists exceptional non-mixing flow in this open set as it was shown by Chaika and Wright [14]. Examples of mild mixing (which is an intermediate property between weak mixing and mixing) were also built in [41] by Kanigowski and Kułaga-Przymus, using the former work [60] of the latter, but again are non typical (and one might conjecture that mild mixing is indeed non typical).

Furthermore, there are also quantitative results on the speed of mixing (when there is mixing) which show that it happens (as expected in a parabolic flow) very slowly. More precisely, for a typical $\varphi_{\mathbb{R}}$ in $\mathscr{U}_{\neg \min }$, restricted to a a minimal component (which is mixing and hence display decay of correlations, refer to Sect. 2 for definitions), the speed of decay of correlations (also sometimes called speed of mixing) is sub-polynomial (in accordance to what we expect for a slowly chaotic flow) and actually logarithmic, namely for every pair $f, g$ of smooth observables there exists constants $c>0, \alpha>0$ such that $\left|C_{f, g}(t)\right| \leq c \log t^{\alpha}$ (as shown by Ravotti in [73]).

\subsection{The role of shearing in slow mixing}

The parabolic nature of locally Hamiltonian flows is entirely due to the presence of the saddles, which split nearby trajectories (as shown in Fig. 6) and are responsible for the slow divergence of nearby trajectories through a geometric phenomenon called shearing (pictured in Fig. 4). The butterfly effect in locally Hamiltonian flows happens indeed in a special way. In presence of a Hamiltonian saddle, the closer a trajectory is to a saddle point, the more motion along the trajectory is slowed down. Thus, if we consider a small arc $\gamma$ transversal to the trajectories of the flow $\varphi_{\mathbb{R}}$, so that when flowing it, $\varphi_{t}(\gamma)$ passes nearby a saddle separatrix without hitting the saddle point (as shown in Fig. 4), the different deceleration rates of points cause $\varphi_{t}(\gamma)$ to shear in the direction of the flow (see Fig. 4a). This description also evidences the slow nature of the butterfly effect in this case: points on nearby trajectories diverge from

shearing estimates and faster mixing. Mixing in this case is indeed believed to have polynomial rates (see e.g. a partial result in this direction in [17]), as opposed to the logarithmic (sub-polynomial) speed in the case of non-degenerate saddles (see [73] and the comments after Theorem 2 in Sect. 3.8).

22 Absence of mixing for typical flows for any $g \geq 2$ was proved in [86], while weak mixing is proved also for minimal components of locally Hamiltonian flow with simple saddles in [85]. Let us remark that a result in this direction for $g=1$ was already proved by Kocergin in [52] (see also [54]) (in the language of special flows over rotations, which does not have a direct implication for locally Hamiltonian flows but suggested that the absence of mixing could hold also when rotations are replaced by IETs and hence in higher genus. Absence of mixing in the special case of $S$ with $g=2$ and locally Hamiltonian flows with two isomorphic simple saddles was shown by Scheglov [78].

23 The result in this generality is proved in [73]. Mixing in a special (but crucial) case was proved in [84] in the language of special flows, more precisely for special flows over interval exchange maps under a roof with one asymmetric logarithmic singularity. The general case is the case of several logarithmic singularities with a global asymmetry condition (see e.g. [73]). 


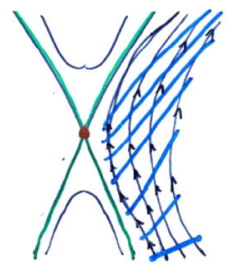

(a) shearing

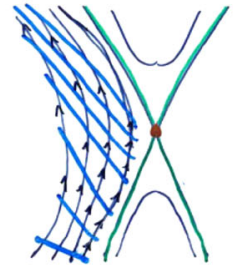

(b) compensation

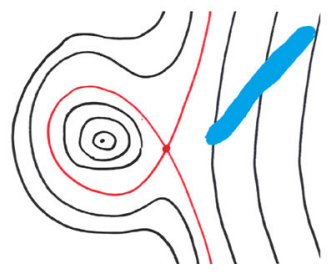

(c) asymmetry

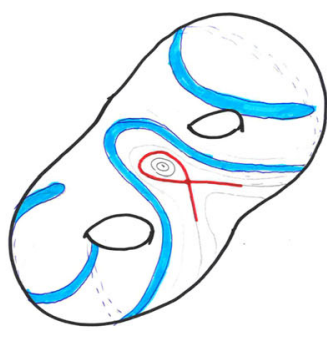

(d) wrapping

Fig. 4 Shearing mechanism in locally Hamiltonian flows

each other since, even though they travel on nearby trajectories, one travels faster than the other. Therefore the speed of divergence is in this case only as fast as the speed of shearing.

The shearing accumulated can be later destroyed when the $\varphi_{t}(\gamma)$ passes near the other side of a saddle (see Fig. 4b). The presence of a saddle loop, though, (as in Fig. 4c) typically creates an asymmetry (this was the key intuition of Arnold that had motivated his conjecture on mixing) by producing stronger shearing on one side and hence, in this case, the accumulation of shearing predominantly in one direction produces global shearing.

This geometric shearing phenomenon is a crucial ingredient in the proofs of the mixing results in Sect. 3.8 (in particular Theorem 2, but also to prove mixing in the exceptional examples in [14]) as it allows to deduce mixing from ergodicity (i.e. equidistribution of flow trajectories, recall Sect. 2). For large times $t \gg 0$, segments which do not hit the singularities will be so sheared along the flow, to be well approximated by long flow trajectories. Thus, for any given measurable set $A \subset X$, for every large $t$ one can cover an arbitrarily large proportion $A_{t} \subset A$ of with a collection of short transversal segments $\left\{\gamma_{\alpha}, \alpha \in \mathscr{A}_{t}\right\}$, each of which, after time $t$, shadows a long trajectory of $\varphi_{\mathbb{R}}$, which is (close to) equidistributed by ergodicity. One can hence show that each $\varphi_{t}\left(\gamma_{\alpha}\right)$ is also (close to) equidistributed. Thus, since

$$
\varphi_{t}\left(A_{t}\right) \cap B=\cup_{\alpha \in \mathscr{A}_{t}}\left(\varphi_{t}\left(\gamma_{\alpha}\right) \cap B\right)
$$

by a Fubini argument, one can deduce equidistribution of $\varphi_{t}(A)$ (i.e. mixing, see Sect. 2) from equidistribution of each $\varphi_{t}\left(\gamma_{\alpha}\right)$ (which follows as we said from shearing and unique ergodicity). Furthermore, the speed of mixing (or equivalently the speed of decay of correlation) depends on the speed of shearing, which is slow (namely subpolynomial in this case).

This mechanism for mixing via shearing seem to be a very common phenomen in slowly chaotic dynamics. The few of the early results on horocycle flows (such as Marcus proof of mixing in [61] or Ratner's results, see Sect. 3.10) exploit that small segments of geodesics curves, pushed by the horocycle flow, are sheared in the horocycle direction. ${ }^{24}$ Furthermore, since this is essentially a geometric mechanism for explaining mixing, this phenomenon persists under perturbation and hence can be used also for time-changes (see $[29,61]$, where we prove quantitative mixing results and show polynomial estimates on the decay of correlations for smooth time-changes of the horocycle flow).

\footnotetext{
24 This shearing property follows from the commuting relations between the horocycle flow $h_{\mathbb{R}}$ and the geodesic flow $g_{\mathbb{R}}$, namely the relation $h_{t} g_{s}=g_{s} h_{e^{-2 s}}$ which holds for all $t, s \in \mathbb{R}$. The relation shows that if we push a small arc $\gamma=\left\{g_{s}(x), s \in[0, \sigma]\right\}$ by $h_{t}$, the point $h_{t}\left(g_{s}(x)\right)$ is aligned in the geodesic direction not with $h_{t}(x)$, but with $h_{e^{-2 s} t}(x)$ and hence that the pushed segments are sheared in the flow direction.
} 


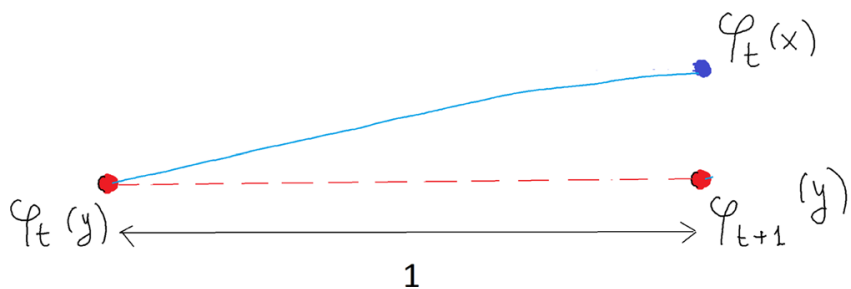

(a) Splitting (shift of 1-time unit) and realignement.

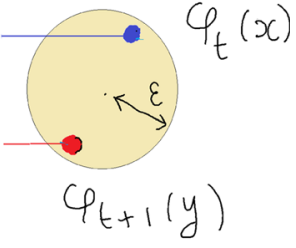

(b) Realigned points stay close

Fig. 5 The Ratner property describing quantitative slow shearing

A similar mechanism, namely shearing of segments of a suitable foliation (but with the difference that the direction of shearing is not global but depends on the segment considered) was also exploited in [20] to prove mixing in some (exceptional) elliptic flows ${ }^{25}$ and in the context of nilflows: while nilflows are never mixing (see footnote 14), in suitable classes of smooth time-changes one can implement this mechanism to prove mixing using shearing, see $[4,5,74]$.

Finally, the complementary results on absence of mixing (see Theorem 1) involve showing absence of shearing. ${ }^{26}$ Indeed, a criterion for absence of mixing already formulated by Kocergin in [52] shows that (at least for typical) locally Hamiltonian flows mixing via shearing is essentially the only possible way of achieving mixing.

\subsection{Beyond mixing, exploting shearing: Ratner's work}

Whether one can deduce stronger and finer ergodic and spectral properties from shearing, in the context of flows with singularities, has been an open problem for decades, which has seen advances only very recently (see Sect. 3.12). A great example of the fine and deep results on finer ergodic properties and rigidity phenomena that one can obtain from shearing is given by the celebrated works by Marina Ratner on the horocycle flow (and more generally unipotent flows in homogeneous dynamics) [68-71]. Her work, and more in general the rigidity theory for unipotent flows, developed by Dani, Margulis and many others, has found breakthrough applications and has led to the solutions of important problems in number theory (such as the Oppenheim conjecture) and mathematical physics (such as the Bolztmann-grad limit for the Lorentz-gas).

Shearing is at the at the heart of Ratner's work and the above mentioned rigidity results. A crucial ingredient in her work, indeed, is a technical property introduced in [70] (that she calls property $H$ ), nowadays known as the Ratner property (see [82]). It is this property, that Ratner verified for horocycle flows, that is used to deduce some of the main rigidity properties of horocycle flows (such as joinings and measure rigidity).

25 In [20], Fayad shows that there exists mixing time-changes of linear flows on tori $\mathbb{T}^{n}=\mathbb{R}^{n} / \mathbb{Z}^{n}$ in dimension $n \geq 3$. The phenomenon is rare though, since it requires a highly Liouvillean rotation number.

26 To show absence of shearing, one needs to exploit that, when there are no saddle loops homologous to zero and hence no asymmetry which produces global shearing, the effect of shearing on two different sides of the same saddles compensate and cancels. This requires subtle estimates which hold for a full measure set of flows in $\mathscr{U}_{\neg \min }$. In the exceptional mixing examples built in [14] sharing is still at the base of mixing, but is not produced by asymmetry of the singularities, but by an asymmetric equidistribution, so that trajectories, at different time scales, spend much more time on one side of a saddle than another) 

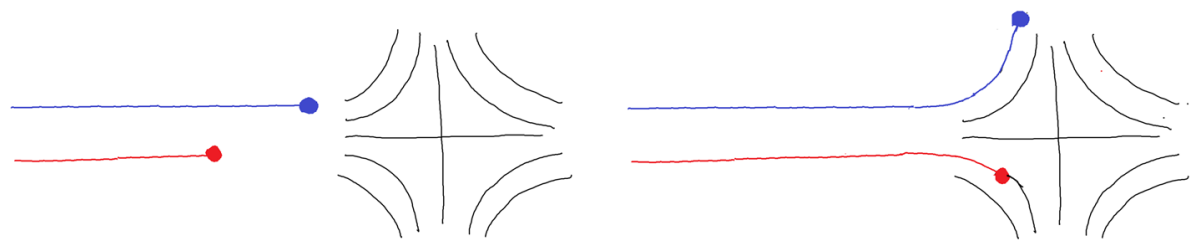

Fig. 6 Splitting of trajectories of a locally Hamiltonian flow near a saddle

The Ratner property encodes a quantitative property of controlled divergence of nearby trajectories in the flow direction (illustrated in Fig. 5). Heuristically, it requires that for most pairs of nearby points $x, x^{\prime}$, the orbits of $x, x^{\prime}$ split in the flow direction (say at time $t_{1}$ ) by a definite amount, called the shift and then realign, say by \pm 1 time-unit ${ }^{27}$ so that now $\varphi_{t_{1}}(x)$ and the time-shifted orbit point $\varphi_{t_{1} \pm 1}\left(x^{\prime}\right)$ are close; then one requires the two orbits, $\left(\varphi_{t_{1}+t}(x)\right)_{t \geq 0}$ and the time-shifted orbit $\left(\varphi_{t+1 \pm 1+t}\left(x^{\prime}\right)\right)_{t \geq 0}$, to still stay close (see Fig. 5) for a fixed proportion $\kappa$ of the time $t_{1}$ it took to see the shift, namely for most times $t \in\left[t_{1}, t_{1}+\kappa t_{1}\right]$. One can see that this type of phenomenon is possible only for parabolic systems, in which orbits of nearby points diverge with polynomial or subpolynomial speed.

\subsection{Searching for Ratner properties beyond unipotent flows}

Since the Ratner property describes a form of divergence of nearby trajectories (or butterfly effect) which is peculiar to parabolic flows, it is reasonable to expect that some quantitative form of parabolic divergence similar to the Ratner property should hold and be crucial in proving analogous rigidity properties for other classes of parabolic flows. Even more, since there is no formal definition for a system to be parabolic, one might even hope that the Ratner property could be taken as one of the characteristics making a system parabolic.

The natural question hence arose whether the Ratner property might hold for smooth flows on surfaces of higher genus. For a long time, though, there were no known examples of systems with the Ratner property beyond horocycle flows and their (smooth) time changes. This changed drastically in the last decade. The first examples outside the homogeneous world were given by Frạczek and Lemańczyk in [33-35] (in the setting of special flows). The two authors could also show in [33] that a variant of Ratner's property hold for some surface flows, more precisely in a class of flows on genus one tori known as von Neumann flows ${ }^{28}$ (for non generic flows, corresponding to a measure zero set of frequencies). However, the flows in [33] are not (globally) smooth.

The difficulty in treating smooth flows on higher genus surfaces is given by the presence of singularities (which are unavoidable when $g \geq 2$, see Sect. 3.2), which introduce discontinuities and destroy the slow form of divergence a la Ratner: essentially, as soon as two nearby trajectories are separated by hitting a saddle (see Fig. 6), one drastically looses control of the divergence. The Ratner property in its classical form (as well as the weaker versions defined

${ }^{27}$ One can more in general consider a shift $p$, which belongs to a fixed compact set $P$ so that now $\varphi_{t+p}(x)$ and the time-shifted orbit $\varphi_{t+p}\left(x^{\prime}\right)$ are close. The original Ratner property, where $P=\{+1,-1\}$, is now sometimes called 2-point Ratner property, while the generalization to $P$ finite first and to $P$ compact later were defined by by Fraczek and Lemańczyk in $[33,34]$ and called respectively finite Ratner and weak Ratner properties.

28 Von Neumann flows are called in this way since they first appeared in von Neumann's work [89]. In the special flows presentation they are flows over irrational rotations under a piecewise linear roof with non zero sum of jumps. For further recent results on von Neumann flows, see [16,43,44]. 
in $[33,34])$ is expected to fail for of smooth area-preserving flows with non-degenerate fixed points. $^{29}$

\subsection{The Switchable Ratner property in locally Hamiltonian flows}

The possibility of pushing our understanding of smooth flows on surfaces, using techniques loosely inspired by Ratner's work, emerged only recently, in virtue of the recent developments in the field. A key breakthrough was achieved recently by Fayad and Kanigowski, who, in [18], introduced a new modification of the Ratner property, the so called Switchable Ratner property (or SR-property). According to this variation, it is sufficient to see the Ratner divergence of orbits for most pairs of initial conditions $(x, y)$ either in the future (for $t>0$ ) $o r$ in the past (for $t<0$ ), depending on the pair of initial points. Thus, if one pair of nearby trajectories is separated by hitting a singularity (as shown in Fig. 6), and hence their distance explodes in an uncontrolled manner, one can still hope to be able to prove the Ratner slow form of divergence when flowing backward in time.

Let us remark that above mentioned variations Ratner property (thus in particular also the switchable Ratner property) were defined in order to have the same strong dynamical consequences of the original Ratner property. In particular, all variants of the Ratner property, as the original Ratner property does, imply a rigidity-type result on joinings ${ }^{30}$ results (by restricting the type of self-joinings that the flow can have ${ }^{31}$ ) and allow to enhance mixing properties (see for example Corollary 1).

Fayad and Kanigowski could prove in [18] that this variation of the Ratner property holds for some smooth surface flows in genus one, more precisely for typical Arnold flows (see Sect. 3.6, Fig. 1b) as well as for (a measure zero class of) torus flows with one degenerate (or fake) singularity (sometimes known as Kocergin flows). Let us recall that in higher genus $(g \geq 2)$ it is important to distinguish between the two open sets $\mathscr{U}_{\text {min }}$ and $\mathscr{U}_{\neg \text { min }}$ (see Sect. 3.7) of locally Hamiltonian flows with non-degenerate singularities. In [41], the SR-property was proved for some (measure zero set of) minimal smooth flows ${ }^{32}$ in $\mathscr{U}_{\text {min }}$. It is likely that to prove a form of Ratner properties for other flows (hopefully a full measure set) in $\mathscr{U}_{\text {min }}$ will

\footnotetext{
29 The failure of the classical Ratner property was formally proved in a special case in [18] (for a class of Kochergin flows, i.e. special flows with power singularities over rotations, see Theorem 1 and the Appendix B in [18]) and this result gives reasons to believe that, for similar reasons, the classical Ratner property should indeed always fail in presence of singularities.

30 The notion of joining plays a key role in ergodic theory. Assume that $\varphi_{\mathbb{R}}, \phi_{\mathbb{R}}$ are flows on probability standard Borel spaces $(X, \mu)$ and $(Y, v)$, respectively. A joining between $\varphi_{\mathbb{R}}$ and $\phi_{\mathbb{R}}$ is a $\varphi_{\mathbb{R}} \times \phi_{\mathbb{R}}$-invariant (for each $t \in \mathbb{R}$ ) probability measure on $X \times Y$ with the projections $\mu$ and $\nu$, respectively. By $J\left(\varphi_{\mathbb{R}}, \phi_{\mathbb{R}}\right)$ we denote the set of joinings between the flows $\varphi_{\mathbb{R}}$ and $\phi_{\mathbb{R}}$. A trivial joining always exists and is given by the product measure $\mu \times \nu$.

31 The Ratner property and its variations in particular imply a property known as finite extension of joinings: any non-trivial ergodic joining $\rho$ is a finite extension (finite union of graphs). Furthermore, Ratner properties impose some restrictions not only on the set of self-joinings but also on the set of its joinings with another (ergodic) flow. In particular, we can ask whether for two systems sharing the same Ratner property it is possible to classify joinings between them. The first result in this direction can be found in Ratner's work [71], where she shows that two flows $\bar{h}_{\mathbb{R}}$ and $\tilde{h}_{\mathbb{R}}$ given by two smooth time changes of a horocycle flow $h_{\mathbb{R}}$ are disjoint, i.e. the only joining between them is the product measure, whenever the cocycles corresponding to the time changes are not jointly cohomologous (see [71] for definitions).

32 The flows considered in [41] are special flows over IETs of bounded type, under a roof with symmetric logarithmic singularities. Bounded type Diophantine conditions on IETs extend the notion of bounded type (also called constant type) rotation numbers and, as among rotations, are measure zero (but full Hausdorff dimension) conditions.
} 
require introducing yet another variant of the Ratner property, one which could take into consideration average shearing and thus will require new ideas.

The result in [41], on the other hand, shows that the switchable Ratner property holds for (the minimal component of) the typical (Arnold) flow in $\mathscr{U}_{\neg \text { min }}$ when $g=1$ and the flow has only one simple saddl (and center). In joint work with Kanigowski and Kułaga-Przymus [42], we could prove that the switchable version of the Ratner property is typical among mixing (components of) locally Hamiltonian flows in $\mathscr{U}_{\neg \text { min }}$ for any genus $g \geq 1$ (thus extending to more singularities ${ }^{33}$ and generalizing to higher genus $g \geq 2$ the result by [18]):

Theorem 3 (Kanigowski, Kułaga-Przymus and U' [42]) For any $g \geq 1$, a typical locally Hamiltonian flow $\varphi_{\mathbb{R}}$ in $\mathscr{U}_{\neg \text { min }}$, restricted on any of its mininal component, has the switchable Ratner form of shearing.

This result hence imply a rigidity type result for the classification of joinings (see footnote 31 ) and in particular allowed us to upgrade mixing to a stronger property, namely mixing of all orders (see Sect. 2 and (4) for the definition).

Corollary 1 (KKU) For any $g \geq 1$, the restriction of a typical locally Hamiltonian flow $\varphi_{\mathbb{R}}$ in $\mathscr{U}_{\neg \text { min }}$ on any of its miminal components is mixing of all orders.

Thus, the Corollary show that Rohlin's conjecture (see Sect. 2) holds for these class of smooth flows.

Further recent works also show that Ratner properties also hold for other classes of slowly chaotic flows. For example the Switchable Ratner property holds for a class of time changes of constant type Heisenberg nilflows, see the recent work [18] by Forni and Kanigowski.

\subsection{Disjointness of rescalings}

Advances in our understanding of disjointness properties became possible building on the switchable Ratner property $[6,19,29]$. The notion of disjointness ${ }^{34}$ was introduced in the 1970s by Furstenberg (see in particular [37]); two disjoint flows are in particular not isomorphic. $^{35}$

A disjointness property which has received a lot of attention recently (in particular as a tool in connection with Sarnak's conjecture on Moebius orthogonality, see below) is disjointness of rescalings. Given a real number $\kappa>0$, by the $\kappa$-rescaling of $\varphi_{\mathbb{R}}$ we simply mean the flow $\varphi_{\mathbb{R}}^{\kappa}:=\left(\varphi_{\kappa t}\right)_{t \in \mathbb{R}}$ (in which the time is rescaled by the factor $\kappa$ ). ${ }^{36}$ Thus, a rescaling is a

\footnotetext{
${ }^{33}$ For $g=1$, Fayad and Kanigowski show in [18] that the switchable Ratner property holds for (the minimal component of) a full measure set of Arnold flows only when there is a unique saddle; they also consider the case of more saddles, but then require a condition which has measure zero. The main result in [42] (which can be expressed in the language of special flows over IETs under roofs with asymmetric logarithmic singularities) on the other hand gives a full measure condition not only the higher genus case, but also the case of genus one and more saddles.

34 Two measure preserving flows $\varphi_{\mathbb{R}}$ and $\phi_{\mathbb{R}}$ are called disjoint (in the sense of Fursterberg) if their only common ergodic joining is the trivial joining (i.e. the product joining).

35 We say that two flows $\varphi_{\mathbb{R}}$ on $(X, \mu)$ and $\phi_{\mathbb{R}}$ on $(Y, v)$ and are isomorphic as measure preserving flows if there exists a isomorphism $\Phi: X \rightarrow Y$, i.e. a one-to-one measureable map which respects measure zero sets and commutes with the dynamics, i.e. $\Phi \circ \phi_{t}(x)=\psi_{t}(\Phi(x))$ for $\mu$-almost every $x \in X$. If two flows are isomorphic, the isomorphism $\Psi$ yields a non-trivial joining, so that the two flows cannot be disjoint.

${ }^{36}$ Notice that when $p$ is an integer, the time-one map of $p$-rescaling coincides with the $p$-power of the time-one map $R_{1}$ of the flow, so considering rescalings is an analogous operation to considering the powers of a given transformation.
} 
special type of time-reparametrization of a flow, given by a linear time-change. We say that $\varphi_{\mathbb{R}}$ has disjoint rescalings if for all (or all but finitely many) $p, q>0$, the rescalings $\varphi_{\mathbb{R}}^{p}$ and $\varphi_{\mathbb{R}}^{q}$, where $p, q>0$ and $p \neq q$, are disjoint (in the sense of Furstenberg). Disjointness of rescalings has played a key role in proving some of the first instances of Sarnak's conjecture [77] of orthogonality of the Moebius function ${ }^{37}$ in number theory with entropy zero dynamical systems (as a tool to prove the conjecture via the so called Katai orthogonality criterion, see for example [8,25] and more in general the survey [21]).

In recent joint work with Kanigowski and Lemańczyk [40], we introduced a new tool to study disjointness phenomena for smooth surface flows, namely a disjointness criterion based on the switchable Ratner property. The criterion was devised and formulated so that it can be applied to prove disjointness of two flows which both have the switchable Ratner property, so that in both flows one can observe a controlled form of divergence of nearby trajectories (for example polynomial divergence), but the speed of divergence for the two flows is different (for example for one flow is it linear, in the other quadratic). ${ }^{38}$ Exploiting this criterium, we were able to show that disjointness of rescalings is typical among Arnold flows (see Sect. 3.6, Fig. 1b).

Theorem 4 (Kanigowski-Lemanczyk-U', [40]) A typical Arnold flow has disjoint rescalings. In particular, if $\varphi_{\mathbb{R}}$ is the restriction of the unique minimal component of an Arnold flow, there exists only two values ${ }^{39}$ of the form $q, 1 / q$ such that $\varphi_{\mathbb{R}}$ and $\varphi_{\mathbb{R}}^{p}$ are disjoint for any positive $p \notin\{1, q, 1 / q\}$.

As a Corollary, Sarnak's Moebius disjointness conjecture holds for these flows (see [40] for details).

We believe disjoitness of rescalings should also hold for typical locally Hamiltonian flows in higher genus, but this is currently an open problem. Preliminary work seems to indicate that, despite some technical additional difficulties, the techniques used to prove Theorem 4 should allow to prove disjointness of rescalings for all minimal components of typical flows in the open set $\mathscr{U}_{\neg \text { min }}$ (refer to Sect. 3.7 for the definition of the open set $\mathscr{U}_{\neg \text { min }}$ ). The recent work [6] by Berk and Kanigowski, even though it does not apply to surface flows directly, gives a good indication that disjointness of rescalings could also hold for typical flows (under a suitable full measure Diophantine-type condition) in the complementary set $\mathscr{U}_{\neg \min }$.

It is natural to ask whether disjoitness of rescalings could actually be a widespread feature of slowly chaotic systems. The new disjointness criterion is also used in [40] to prove disjointness of rescalings for (a class of) smooth (non-trivial) time-changes of the horocycle flow (see also [25], where the result is proved for a more general class of time changes with different methods), answering in particular a question of Marina Ratner. Notice that

\footnotetext{
${ }^{37}$ Let us recall that the Moebius function $\mu: \mathbb{N} \rightarrow \mathbb{Z}$ is a multiplicative function defined on a prime $p$ by $\mu(p):=(-1)^{k}$ if $p=p_{1} \cdots p_{k}$ with $p_{i}$ istinct primes. Sarnak conjectured (see [77]) that $\mu$ is orthogonal to entropy zero deterministic sequences, i.e. if $f: X \rightarrow X$ is a topological dynamical systems (i.e. $X$ is a topological space and $f$ is continous) then, for every $x \in X, \sum_{n=1}^{N} f\left(T^{n} x\right) \mu(n)=o(N)$ as $N$ tends to infinity. The survey [21] gives an introduction to the conjectures and the progress made so far.

38 The key shearing phenomenon exploited in the criterion is that, for pairs of two nearby points in the first system and two nearby points in the second, after some time (depending on both pairs of points) we will see a relative divergence, i. e. in one pair we will see a realignement with some shift $C_{1}>0$ in the flow direction, while in the other we will see a realignement with a shift $C_{2}>0$ with $C_{1} \neq C_{2}$. This explains how the relative shearing appears in this situation.

39 The two values are related to the asymmetry of the saddles: in the special flow representation, the Arnold flow can be written as a flow over a rotation $R_{\alpha}:[0,1) \rightarrow[0,1)\left(\right.$ given by $\left.R_{\alpha}(x)=x+\alpha \bmod 1\right)$ under a roof with logarithmic singularities given by the roof function $r(x)=C_{0} \log |x|+C_{1} \log (1-x)+h(x)$ where $h$ is smooth on $[0,1]$; then $q=C_{0} / C_{1}$.
} 
two different rescalings of the (classical, non time-changed) horocycle flow $h_{\mathbb{R}}$ are never disjoint. ${ }^{40}$ Thus, it seems that (non trivial) time-changes of the horocycle flow are in some sense better behaved and display chaotic features that the horocycle flow itself lacks, due to its homogeneous and self-similar nature. Perhaps unfortunately, the most studied and best understood model of a parabolic flow, the horocycle flow, may have not provided the most significant example in terms of generic chaotic properties. Hence, the importance of better understanding new and larger classes of slowly chaotic systems and their typical chaotic features.

The new criterion for disjointness introduced in [40] has already proved useful in different contexts, see for example the recent works [16,28] where it is applied to study disjointess phenomena respectively for Heisenberg nilflows in [28], for von Neumann flows in genus one in [16]. Finally, the disjointness criterion is used in [40] also to show that a typical Arnold flow is disjoint from any smooth time change of the horocycle flow (and in particular from the classical horocycle flow itself), thus showing that these two classes of parabolic flows are truly distinct.

\subsection{Spectral theory of locally Hamiltonian flows}

The last aspect we want to discuss is the spectral theory of smooth area-preserving flows (introduced in Sect. 2). The spectral properties (and in particular what is the spectral type, see Sect. 3.14 for definitions) of locally Hamiltonian flows is a natural question, which has been lingering for decades (see e.g. [49, Section 6] and [58]). While the classification of mixing properties of locally Hamiltoninan flows is essentially complete (as summarized in Sect. 3.8), very little is known on the spectral properties of these flows beyond the case of genus one.

One of the few results in the literature concerning on the nature of the spectrum of some area-preserving surface flows was proved by Frączek and Lemańczyk in [32], who showed singularity of the spectrum for Blokhin examples ${ }^{41}$ (which were the first examples of ergodic flows on surfaces, see [7]). This gives examples of locally Hamiltonian flows on surfaces of any genus $\geq 1$ with singular continuous spectrum (see [30, Theorem 1]), but these are essentially built glueing genus one flows and thus, they are highly non typical.

It turns out that the geometric approach to (quantitative) mixing through shearing can sometimes be pushed to provide also spectral information on parabolic flows. For example, in joint work with Forni [29] we were able to show that from shearing estimates on can also access information about the spectrum of time-changes of the horocycle flow, in particular settling in particular a conjecture of Katok-Thouvenot (see also [83] where Tiedra de Aldecoa gave simultaneously a different proof by operator methods (see also a previous result by Kuschnirenko [61]). Further developments based on our approach were achieved also for unipotent flows by Simonelli [79].

A breakthrough on the spectral side was achieved recently in [19] for a special class of smooth area preserving flows with degenerate singularities when the genus of the underlying

40 Indeed, if $\left(g_{s}\right)$ denotes the geodesic flow, the renormalization equation $h_{t} g_{s}=g_{s} h_{e}-2 s_{t}$ for all $t, s \in \mathbb{R}$, yields that, for any positive $p \neq q$, the flows $h_{\mathbb{R}}^{p}$ and $h_{\mathbb{R}}^{q}$ are conjugated by $g_{s}$ with $s=-\frac{\log (q / p)}{2}$ (and hence are not disjoint).

41 In the setting of special flows, Frạczek and Lemańczyk in [30], study special flows over rotations with single symmetric logarithmic singularity. In [30, Theorem 12] it is shown that (for a full measure set of rotation numbers) such special flows are spectrally disjoint from all mixing flows, from which it follows in particular that the spectrum is purely singular. 


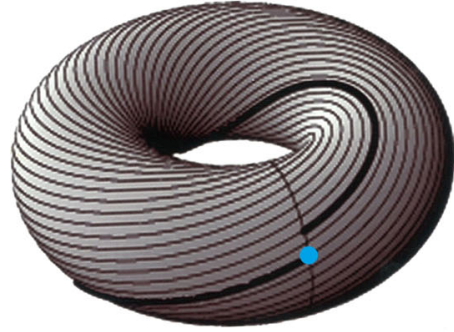

(a) Kochergin flow (a.c.spectrum)

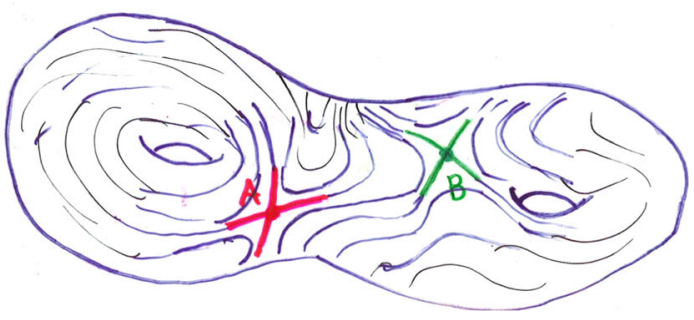

(b) $\mathrm{g}=2$, two isomorphic simple saddles (singular)

Fig.7 The locally Hamiltonian flows with absolutely continuous and singular spectra respectively in Theorems 5 and 6

surface is one, sometimes known as Kochergin flows (since Kochergin [53] proved their mixing, for any $g \geq 1$ ). These are minimal flows on the torus with one stopping point (see Fig. 7a), also called fake singularity (this point can be seen as a degenerate fixed point with only $k=2$ prongs). Taking as a starting point the idea used by Forni an myself in [29] to prove absolute continuity of the spectrum for time changes of horocycle flows, Forni, Fayad and Kanigowski, proved in [20] that, if the degenerate singularity is sufficiently strong ${ }^{42}$, the spectrum is absolutely continuous (and actually Lebesgue).

Theorem 5 (Fayad, Forni, Kanigowski, [19]) A locally Hamiltonian flow in genus one with only one sufficiently strong degenerate singularity as fixed point has countable Lebesgue spectrum.

Countable Lebesgue spectrum is a strong spectral result, which implies in particular that the spectrum is absolutely continuous (see Sect. 2 for the definition). The result provides the first example of such a strong chaotic property in an entropy zero and low dimensional smooth system. We remark that stopping points (and more in general degenerate fixed points) are known to produce shearing and hence mixing [53] (at rates which are expected to be polynomial, see e.g. [17]). The absolute continuity of the spectrum is essentially ${ }^{43}$ based on a decay of correlations which is square-summable.

A recent spectral breakthrough, which goes in the opposite direction, concerns the nature of the spectrum of locally Hamiltonian flows on genus two surfaces, and, to the best of our knowledge, is the first general spectral result for surfaces of higher genus, namely $g \geq 2$.

Theorem 6 (Chaika-Fraczek-Kanigowski-U', [13]) A typical locally Hamiltonian flow on a genus two surface with two isomorphic simple saddles has purely singular spectrum.

This result in genus two was inspired by the singularity result proved by Fraczek and Lemańczyk (for special flows over rotations) in [30]. Their result indeed shows that, when one can prove absence of mixing and some form of (partial) rigidity, it might be possible to deduce singularity of the spectrum. Theorem 6 strengthens one of the early results on absence of mixing, i.e. the absence of mixing for typical flows in the same class ( $g=2$, two isomorphic saddles) proved by Scheglov [78] (which is a special case of Theorem 1). As in

42 Kocergin flows admit a special flow representation where the roof has power-type singularities. In genus one, for a flow with one degenerate singularity, one has a special flow over a rotation, under the roof $r(x)=$ $c_{0} / x^{\alpha}+c_{1} /(1-x)^{\alpha}$ for some $0<\alpha<1$. The assumption in [19] is that $\alpha$ is sufficiently close to 1 .

43 There are actually technical issues in controlling the part of space where there is not enough shearing. 
[78], the assumptions are crucial since the underlying surface has an inner symmetry ${ }^{44}$ which plays a crucial role in the proof. Nevertheless, we believe it should be possible to extend the result on higher genus exploiting the same singularity criterion used in [13] (which is an extension of the criterion used in [30] as well as [31]), coupled with the delicate estimates for absence of shearing proved in [86] to prove Theorem 1 .

The nature of the spectrum for other classes of locally Hamiltonian flows is unknown. It might be conjectured, in view of Theorem 5 for Kocergin flows, that also in higher genus, in presence of sufficiently strong degenerate singular points, the spectrum is also absolutely continuous (and even countable Lebesgue), essentially thanks to a strong quantitative control of decay of correlations. It is not clear what to expect when the degenerate singularity is not sufficiently strong. ${ }^{45}$

At the heart of our proof of Theorem 6, on the other hand, is a strengthening of results on absence of mixing (in particular of the works [30,31,78]). As already mentioned, we hope that the techniques of [86] might be pushed to allow to apply the singularity criterium for typical flows in the open set $\mathscr{U}_{\text {min }}$ of minimal, uniquely ergodic (weakly mixing) but not mixing locally Hamiltonian flows.

In the open set $\mathscr{U}_{\neg \text { min }}$, which consists of flows with non-degenerate singularities that are not minimal, but have several minimal components, the nature of the spectrum (for the restriction of a typical flow to a minimal component) is unclear. These flows are indeed mixing, but with sub-polynomial rate (see [73], which provides logarithmic upper bounds) and it is not clear whether to expect singularity or absolute continuity of the spectrum.

Acknowledgements The author is part of SwissMAP (The Mathematics of Physics National Centre for Compentence in Research) and is currently supported by a SNSF (Swiss National Science Foundation) Grant No. 200021_188617/1. Both are acknowledged for their support.

Funding Open access funding provided by University of Zurich.

Open Access This article is licensed under a Creative Commons Attribution 4.0 International License, which permits use, sharing, adaptation, distribution and reproduction in any medium or format, as long as you give appropriate credit to the original author(s) and the source, provide a link to the Creative Commons licence, and indicate if changes were made. The images or other third party material in this article are included in the article's Creative Commons licence, unless indicated otherwise in a credit line to the material. If material is not included in the article's Creative Commons licence and your intended use is not permitted by statutory regulation or exceeds the permitted use, you will need to obtain permission directly from the copyright holder. To view a copy of this licence, visit http://creativecommons.org/licenses/by/4.0/.

\section{References}

1. Arnold, V.I.: Topological and ergodic properties of closed 1-forms with incommensurable periods. Funktsional'nyi Analiz i Ego Prilozheniya 25, 1-12 (1991)

2. Auslander, L., Green, L., Hahn, F.: Flows on Homogeneous Spaces. Princeton University Press, Princeton (1963)

3. Avila, A., Forni, G.: Weak mixing for interval exchange transformations and translation flows. Ann. Math. (2) $\mathbf{1 6 5}(2), 637-664(2007)$

\footnotetext{
44 More precisely, the linear flow of which the locally Hamiltonian flow is a time-change is a flow on a translation surface $S$ which admits an hyperelliptic involution, i.e. an affine automorphism $\Phi: X \rightarrow X$ which is an involution, i.e. $\Phi^{2}=I d$.

45 As already remarked in a footnote, sufficiently strong means that the power $\alpha$ in the special flow representation is close to 1 . One might hope that absolute continuity could hold for all powers $\alpha>1 / 2$, but this is out of reach with the current techniques.
} 
4. Avila, A., Forni, G., Ulcigrai, C.: Mixing for time-changes of Heisenberg nilflows. J. Differ. Geom. 89(3), 369-410 (2011)

5. Avila, A., Forni, G., Ravotti, D., Ulcigrai, C.: Mixing for smooth time-changes of general nilflows. arXiv: 1905.11628

6. Berk, P., Kanigowski, A.: Spectral disjointness of rescalings of some surface flows. arXiv:1901.04724v2

7. Blohin, A.A.: Smooth ergodic flows on surfaces. Trudy Moskov. Mat. Obsc. 27, 113-128 (1972). (Russian)

8. Bourgain, J., Sarnak, P., Ziegler, T.: Disjointness of Möbius from horocycle flows. arXiv:1110.0992

9. Bufetov, A., Sinai, Y., Ulcigrai, C.: A condition for continuous spectrum of an interval exchange transformation. In: Representation Theory, Dynamical Systems, and Asymptotic Combinatorics, Amer. Math. Soc. Transl. Ser. 2, 217, Adv. Math. Sci., vol. 58, pp. 23-35. Amer. Math. Soc., Providence (2006)

10. Bufetov, A., Solomyak, B.: The Hölder property for the spectrum of translation flows in genus two. Isr. J. Math. 223(1), 205-259 (2018)

11. Bufetov, A., Solomyak, B.: On the modulus of continuity for spectral measures in substitution dynamics. Adv. Math. 260, 84-129 (2014)

12. Burger, M.: Horocycle flow on geometrically finite surfaces. Duke Math. J. 61, 779-803 (1990)

13. Chaika, J., Fraczek, K., Kanigowski, A., Ulcigrai, C.: Singularity of the spectrum for smooth areapreserving flows in genus two and translation surfaces well approximated by cylinders. arXiv:1912.10250

14. Chaika, J., Wright, A.: A smooth mixing flow on a surface with nondegenerate fixed points. J. Am. Math. Soc. 32(1), 81-117 (2019)

15. Dani, S.G.: Invariant measures and minimal sets of horospherical flows. Invent. Math. 64(2), 357-385 (1981)

16. Dong, C., Kanigowski, A.: Rigidity of a class of smooth singular flows on $T^{2}$. arXiv: 1811.00184

17. Fayad, B.: Polynomial decay of correlations for a class of smooth flows on the two torus. Bull. Soc. Math. France 129, 487-503 (2001)

18. Fayad, B., Kanigowski, A.: Multiple mixing for a class of conservative surface flows. Invent. Math. 1-60 (2015)

19. Fayad, B.R., Forni, G., Kanigowski, A.L.: Lebesgue spectrum for area preserving flows on the two torus. arXiv: 1609.03757

20. Fayad, B.R.: Analytic mixing reparametrizations of irrational flows. Ergod. Theory Dyn. Syst. 22, 437468 (2002)

21. Ferenczi, S., Kułaga-Przymus, J., Lemańczyk, M.: Sarnak’s conjecture: what's new. In: Ferenczi, S., Kuł aga-Przymus, J., Lemańczyk, M. (eds.) Ergodic Theory and Dynamical Systems in their Interactions with Arithmetics and Combinatorics, Lecture Notes in Mathematics, vol. 2213, p. 418. Springer International Publishing

22. Flaminio, L., Forni, G.: Invariant distributions and time averages for horocycle flows. Duke Math. J. 119(3), 465-526 (2003)

23. Flaminio, L., Forni, G.: Equidistribution of nilflows and applications to theta sums. Ergod. Theory Dyn. Syst. 26(2), 409-433 (2006)

24. Flaminio, L., Forni, G.: On the cohomological equation for nilflows. J. Mod. Dyn. 1, 37-60 (2007)

25. Flaminio, L., Forni, G.: Orthogonal powers and Möbius conjecture for smooth time changes of horocycle flows. arXiv:1811.04652

26. Flaminio, Frączek, K., Kułaga-Przymus, J., Lemańczyk, M.: Approximate orthogonality of powers for ergodic affine unipotent diffeomorphisms on nilmanifolds. Stud. Math. 244(1), 43-97 (2019)

27. Forni, G.: Solutions of the cohomological equation for area-preserving flows on compact surfaces of higher genus. Ann. Math. 146(2), 295-344 (1997)

28. Forni, G., Kanigowski, A.: Mutliple mixing and disjointness for time changes of bounded-type Heisenberg nilflows. arXiv: 1810.13319

29. Forni, G., Ulcigrai, C.: Time changes of horocycle flows. J. Mod. Dyn. 6(2), 251-273 (2012)

30. Fraczek, K., Lemańczyk, M.: On symmetric logarithm and some old examples in smooth ergodic theory. Fund. Math. 180(3), 241-255 (2003)

31. Fraczek, K., Lemańczyk, M.: On disjointness properties of some smooth flows. Fund. Math. 185(2), 117-142 (2005)

32. Fraczek, K., Lemańczyk, M.: A class of special flows over irrational rotations which is disjoint from mixing flows. Ergod. Theory Dyn. Syst. 26, 1-21 (2006)

33. Fraczek, K., Lemańczyk, M.: On mild mixing of special flows over irrational rotations under piecewise smooth functions. Ergod. Theory Dyn. Syst. 26, 719-738 (2006)

34. Fraczek, K., Lemańczyk, M., Lesigne, E.: Mild mixing property for special flows under piecewise constant functions. Discrete Contin. Dyn. Syst. 19, 691-710 (2007)

35. Fraczek, K., Lemańczyk, M.: Ratner's property and mild mixing for special flows over two-dimensional rotations. J. Mod. Dyn. 4(4), 609-635 (2010) 
36. Furstenberg, H.: The unique ergodicity of the horocycle flow. In: Recent Advances in Topological Dynamics (New Haven, Conn., 1972), Lecture Notes in Math., vol. 318, pp 95-115. Springer, Berlin (1973)

37. Furstenberg, H.: Reurrence in Ergodic Theory and Combinatorial Number Theory. Princeton University Press, Princeton (1981)

38. Gurevich, B.M.: The entropy of horocycle flows. Dokl. Akad. Nauk SSSR 136, 768-770 (1961). (Russian)

39. Hedlund, G.A.: Fuchsian groups and transitive horocycles. Duke Math. J. 2, 530-542 (1936)

40. Kanigowski, A., Lemańczyk, M., Ulcigrai, C.: On disjointness properties of some parabolic flows. Invent. Math. 221(1), 1-111 (2020)

41. Kanigowski, A., Kułaga-Przymus, J.: Ratner's property and mild mixing for smooth flows on surfaces. Ergod. Theory Dyn. Syst. 36(8), 2512-2537 (2016)

42. Kanigowski, A., Kułaga-Przymus, J., Ulcigrai, C.: Ratner's property and mild mixing for smooth flows on surfaces. J. Eur. Math. Soc. 21(12), 3797-3855 (2019)

43. Kanigowski, A., Solomko, A.: On isomorphism problem for von Neumann flows with one discontinuity. Isr. J. Math. 226(2), 685-702 (2018)

44. Kanigowski, A., Solomko, A.: On the rank of von Neumann special flows. Ergod. Theory Dyn. Syst. 38(6), 2245-2256 (2018)

45. Katok, A.B.: Invariant measures of flows on oriented surfaces. Sov. Math. Dokl. 14, 1104-1108 (1973)

46. Katok, A.B.: Interval exchange transformations and some special flows are not mixing. Isr. J. Math. 35(4), 301-310 (1980)

47. Katok, A.B.: Cocycles, cohomology and combinatorial constructions in ergodic theory (in collaboration with E. A. Robinson, Jr. In: Smooth Ergodic Theory and its applications, Proc. Symp. Pure Math., vol. 69, pp.107-173 (2001)

48. Katok, A.B.: Combinatorial Constructions in Ergodic Theory and Dynamics. University Lecture Series, vol. 30. American Mathematical Society, Providence (2003)

49. Katok, A.B., Thouvenot, J.-P.: Spectral Properties and Combinatorial Constructions in Ergodic Theory. In: Katok, A., Hasselblatt, B. (eds.) Handbook of Dynamical Systems, Vol 1B. Elsevier (2005)

50. Keane, M.: Interval exchange transformations. Math. Zeitschrift 141(1), 25-31 (1975)

51. Khanin, K.M., Sinai, Ya. G... Mixing for some classes of special flows over rotations of the circle. Funktsional'nyi Analiz i Ego Prilozheniya 26(3), 1-21 (1992) (Translated in: Functional Analysis and its Applications, 26:3:155-169, 1992)

52. Kočergin, A.V.: The absence of mixing in special flows over a rotation of the circle and in flows on a two-dimensional torus. Dokl. Akad. Nauk SSSR 205, 512-518 (1972) (Translated in: Soviet Math. Dokl. 13 (1972), 949-952)

53. Kočergin, A.V.: Mixing in special flows over a shifting of segments and in smooth flows on surfaces. Mat. Sb. 96(138), 471-502 (1975)

54. Kočergin, A.V.: Nondegenerate saddles and the absence of mixing in flows on surfaces. Proc. Steklov Inst. Math. 256(1), 238 (2007)

55. Kułaga, J.: On the self-similarity problem for smooth flows on orientable surfaces. Ergod. Theory Dyn. Syst. 32(5), 1615-1660 (2012)

56. Kushnirenko, A.G.: Spectral properties of some dynamical systems with polynomial divergence of orbits. Vestnik Moskovskogo Universiteta. Matematika 29(1), 101-108 (1974)

57. Ledrappier, F., Sarig, O.: Unique ergodicity for non-uniquely ergodic horocycle flows. Discrete Contin. Dyn. Syst. 16, 411-433 (2006)

58. Lemańczyk, M.: Spectral theory of dynamical systems. Mathematics of complexity and dynamical systems, vols. 1-3, pp. 1618-1638, Springer, New York (2012)

59. Levitt, G.: La decomposition dynamique et la differentiabilie des feuilletages des surfaces. Ann. Inst. Fourier 3, 85-116 (1987)

60. Maier, A.: On trajectories on orientable surfaces. Dokl. Acad. Nauk SSSR 24, 672-674 (1939)

61. Marcus, B.: Ergodic properties of horocycle flows for surfaces of negative curvature. Ann. Math. 2(105), 81-105 (1977)

62. Marcus, B.: The horocycle flow is mixing of all degrees. Invent. Math. 46, 201-209 (1978)

63. Masur, H.: Interval exchange transformations and measured foliations. Ann. Math. 115, 169-200 (1982)

64. Nikolaev, I., Zhuzhoma, E.: Flows on 2-dimensional manifolds. An overview, Lecture Notes in Mathematics, vol. 1705. Springer, Berlin (1999)

65. Novikov, S.P.: The Hamiltonian formalism and a multivalued analogue of Morse theory. Uspekhi Matematicheskikh Nauk 37(5), 3-49 (1982) (Traslated in: Russian Mathematical Surveys, 37(5):1-56, 1982)

66. Parasyuk, O.S.: Flows of horocycles on surfaces of constant negative curvature. Uspekhi Mat. Nauk 8(3), $125-126(1953)$

67. Poincaré, H.: Sur les courbes definies par les equations differentielles. J. Math. Pures Appl. 2, 151-217 (1886) 
68. Ratner, M.: Factors of horocycle flows. Ergod. Theory Dyn. Syst. 2, 465-489 (1982)

69. Ratner, M.: Rigidity of horocycle flows. Ann. Math. 2(115), 597-614 (1982)

70. Ratner, M.: Horocycle flows joinings and rigidity of products. Ann. Math. 2(118), 277-313 (1983)

71. Ratner, M.: Rigid reparametrizations and cohomology for horocycle flows. Invent. Math. 88(2), 341-374 (1987)

72. Ratner, M.: The rate of mixing for geodesic and horocycle flows. Ergod. Theory Dyn. Syst. 7, 267-288 (1987)

73. Ravotti, D.: Quantitative mixing for locally Hamiltonian flows with saddle loops on compact surfaces. Ann. H. Poincaré 18 (12), 3815-3861

74. Ravotti, D.: Mixing for suspension flows over skew-translations and time-changes of quasi-abelian filiform nilflows. Ergod. Theory Dyn. Syst. 39(12), 3407-3436 (2019)

75. Ravotti, D.: Parabolic perturbations on unipotent flows on compact quotients of SL(3, $\mathbb{R})$. Commun. Math. Phys. 371(1), 331-351 (2019)

76. Sarnak, P.: Asymptotic behavior of periodic orbits of the horocycle flow and Eisenstein series. Commun. Pure Appl. Math. 34, 719-739 (1981)

77. Sarnak, P.: Three lectures on the Moebius function randomness and dynamics. http://publications.ias.edu/ sarnak/

78. Scheglov, D.: Absence of mixing for smooth flows on genus two surfaces. J. Mod. Dyn. 3(1), 13-34 (2009)

79. Simonelli, L.: Absolutely continuous spectrum for parabolic flows/maps. Discrete Contin. Dyn. Syst. 38(1), 263-292 (2018)

80. Starkov, A.: Dynamical Systems on Homogeneous Spaces, vol. 190. Translations of the American Mathematical Society, Providence (2002)

81. Strömbergsson, A.: On the deviation of ergodic averages for horocycle flows. J. Mod. Dyn. 7(2), 291-328 (2013)

82. Thouvenot, J.P.: Some properties and applications of joining tranformations. J. Mod. Dyn. 3(1), 35-49 (2009)

83. Tiedra de Aldecoa, R.: Spectral analysis of time-changes of the horocycle flow. J. Mod. Dyn. 6, 275-285 (2012)

84. Ulcigrai, C.: Mixing for suspension flows over interval exchange tranformations. Ergod. Theory Dyn. Syst. 27(3), 991-1035 (2007)

85. Ulcigrai, C.: Weak mixing for logarithmic flows over interval exchange tranformations. J. Mod. Dyn. 3(1), 35-49 (2009)

86. Ulcigrai, C.: Absence of mixing in area-preserving flows on surfaces. Ann. Math. 173(2), 1743-1778 (2011)

87. Veech, W.: Gauss measures for transformations on the space of interval exchange maps. Ann. Math. 115, 201-242 (1982)

88. Veech, W.: The metric theory of interval exchange transformations I. Generic spectral properties. Am. J. Math. 106(6), 1331-1359 (1984)

89. von Neumann, J.: Zur Operatorenmethode in der klassischen Mechanik. Ann. Math. 33, 587-642 (1932)

90. Young, L.S.: Entropy of continuous flows on compact 2-manifolds. Topology 16(4), 465-467 (1977)

91. Zorich, A.: How do the leaves of a closed 1-form wind around a surface? Am. Math. Soc. 197, 135-178 (1999)

Publisher's Note Springer Nature remains neutral with regard to jurisdictional claims in published maps and institutional affiliations. 